\title{
Analysis of ultra-narrow ferromagnetic domain walls
}

\author{
C.A. Jenkins ${ }^{1,2}$ \\ present affiliation: ${ }^{1}$ Advanced Light Source, Lawrence Berkeley National Laboratory, Berkeley, CA 94720 \\ D.I. $\mathrm{Paul}^{2}$ \\ ${ }^{2}$ Department of Materials Science and Engineering, \\ Massachusetts Institute of Technology, Cambridge, MA, 02142
}

(Dated: January 10, 2012)

\begin{abstract}
New materials with high magnetic anisotropy will have domains separated by ultra-narrow ferromagnetic walls with widths on the order of a few unit cells, approaching the limit where the elastic continuum approximation often used in micromagnetic simulations is accurate. The limits of this approximation are explored, and the static and dynamic interactions with intrinsic crystalline defects and external driving fields are modeled. The results developed here will be important when considering the stability of ultra-high-density storage media.

PACS numbers: TBD
\end{abstract}

\section{DISCLAIMER}

This document was prepared as an account of work sponsored by the United States Government. While this document is believed to contain correct information, neither the United States Government nor any agency thereof, nor the Regents of the University of California, nor any of their employees, makes any warranty, express or implied, or assumes any legal responsibility for the accuracy, completeness, or usefulness of any information, apparatus, product, or process disclosed, or represents that its use would not infringe privately owned rights. Reference herein to any specific commercial product, process, or service by its trade name, trademark, manufacturer, or otherwise, does not necessarily constitute or imply its endorsement, recommendation, or favoring by the United States Government or any agency thereof, or the Regents of the University of California. The views and opinions of authors expressed herein do not necessarily state or reflect those of the United States Government or any agency thereof or the Regents of the University of California. 


\section{INTRODUCTION}

Ferromagnetic domain walls are fundamental entities in the development of magnetic recording media, and ultranarrow walls of only a few lattice constants in width are of increasing relevance as processing technologies such as molecular beam epitaxy, self-assembly, and nanolithography gain ever more control over abrupt atomic discontinuities. Micromagnetic simulations [1] treat the magnetized material as an elastic continuum, ignoring locally inhomogeneous atomic contributions to the energetic potential. Density-functional-theoretical approaches miss more macroscopic, non-periodic features such as domain walls as a result of their periodic unit cell definition. This disconnect between atomistic and continuum treatments motivated previous analytical work on crystallographic twin boundaries [2-4], using summation rather than integration in the mathematical formulation to account for the discreteness of the atomic lattice.

This work considers the effects of discreteness on ultra-narrow but purely magnetic boundaries, i.e. with no associated structural distortion. There are three main divisions: first, the intrinsic interatomic potential of a narrow domain wall is calculated to quantify the effect of a periodic atomic impedance to wall motion; second, the interaction of such a narrow domain wall with a similarly narrow planar defect is analyzed to find self-consistent static solutions leading to a coercive force; finally, time dependence is explored by adding an ad hoc pseudo-viscosity (true viscosity being an average macroscopic response) to account for the observed damping.

\section{MODELING AND ASSUMPTIONS}

The direction of the atomic magnetic moment is modeled in most cases to be the local solution to the elastic continuum approximation, conformationally analogous to that of a traveling soliton [5]. The degree of approximation inherent in this method is explored here. We show that in domain walls of width $\pi \delta$ equal to about $9 l$, where $\delta$ is a function of the ratio of the exchange energy to the anisotropy and $l$ is the lattice spacing, the idealized picture of matter as having a uniform potential begins to break down. This is mathematically represented by a change from integration to summation over the atomic energies. The geometry has one-dimensional functional variance in space but models a three-dimensional crystal by assuming a $180^{\circ}$ plane wall. This is the situation in thin films where the anisotropy is uniaxial and the following equations are determined only by boundary conditions.

It was shown in Quattrochi and Paul [6] that the contribution to the energy from the change in anisotropy direction is large when compared with the intrinsic energy or the energy from the material defect. Therefore, in order to isolate the effect of the narrowness of the wall, no rotation in anisotropy axis is permitted. The possibility of multiple walls has also been discounted since a domain wall, unlike a dislocation, can be stable without a complement of opposite "handedness" [7]. For the time-dependent analysis, modified perturbation theory was applied as in previous work by Paul $[8]$.

In defining the dynamic physical problem, one encounters the dilemma of whether to give the discrete lattice an intrinsic stiffness or to define the wall as possessing an intrinsic mass. Döring [9] proposed that the mass of the wall was inversely proportional to its thickness, which suggests that at least for a narrow wall, the mass therein dominates 
TABLE I: Material parameters for the materials under consideration. The unit system is cgs, in deference to magneticians. The relevant conversions are $10 \mathrm{ergs} / \mathrm{cm}^{3}=1 \mathrm{~J} / \mathrm{m}^{3}, 10^{4} \mathrm{Oe}=1 \mathrm{~T} . A$ is the exchange energy, linearly related to the Curie temperature. $K$ is properly $K_{1}$, the anisotropy energy in the easy direction, to some degree declaring each material to be cubic by declaring the $K$ to be isotropic. The units of $M$ are such that $M H \cos \theta$ has units of energy density. Real material data selected from [12] and hypothetical materials 'Future 1' and 'Future 2' have parameters chosen to be consistent with reasonable predictions of available engineered nanomaterials.

\begin{tabular}{lcccccc}
\hline \hline & $\mathrm{SmCo}_{5}$ & $\mathrm{Sm}_{2} \mathrm{Co}_{17}$ & $\mathrm{Nd}_{2} \mathrm{Fe}_{14} \mathrm{~B}$ & $\mathrm{Fe}$ & Fut. 1 & Fut. 2 \\
\hline $\mathrm{A}[\mathrm{erg} / \mathrm{cm}]$ & $1.9 \times 10^{-6}$ & $2.1 \times 10^{-6}$ & $1.1 \times 10^{-6}$ & $2.0 \times 10^{-6}$ & $1.5 \times 10^{-6}$ & $1.3 \times 10^{-6}$ \\
$\mathrm{~K}\left[\mathrm{erg} / \mathrm{cm}^{3}\right]$ & $1.7 \times 10^{8}$ & $3.3 \times 10^{7}$ & $4.9 \times 10^{7}$ & $4.8 \times 10^{5}$ & $5.0 \times 10^{8}$ & $3.0 \times 10^{8}$ \\
$l\left[10^{-8} \mathrm{~cm}\right]$ & 5.0 & 8.4 & 8.8 & 2.9 & 7.0 & 5.0 \\
$\mathrm{M}$ & 700 & 1030 & 700 & 1200 & 800 & 800 \\
\hline
\end{tabular}

any inertial forces from the lattice. It should also be noted that since the inertial term is dependent upon the kinetic energy, there is an implicit temperature dependence in the equations presented.

The interatomic potential was chosen to be sinusoidal for simplicity as Egami and Graham [10], while maintaining to a large extent the detail seen in models employing the double-well potential of Krumhansl and Schrieffer [11].

Table I presents material parameters used in all calculations. Hypothetical materials Future 1 and Future 2 were deemed to have parameters that were selected to be both self-consistent and plausible for engineered materials. Given the fact that the magnitude of the exchange constant should be linearly related to $T_{C}[13]$ and that the $T_{C}$ of our assumed materials should be similar to known metals, exchange constants were chosen near the mean value of the real materials under consideration. Coercivities of two and three times currently achievable values in single-phase $\mathrm{SmCo}_{5}$ were taken as plausible, although whether the material is an engineered heterostructure or a single-phase epitaxial crystal is left open. The coefficient of kinetic energy for the time-dependent analysis is taken for all materials to be $6 \times 10^{9}$, as a compromise between the estimates of Enz [14] and Döring [9].

The un-subscripted $K$ parameter for the anisotropy in units of energy density is the generalized $K_{1}$ from cubic unit cells. According to Neumann's principle, materials properties (including magnetocrystalline anisotropy) have to have at least the symmetry of the material itself. This is written symbolically as a series representing the symmetry, of which the $K_{n}$ are the fitting parameters. However, when considering only uniaxial anisotropy, the higher-order numbers reported record not an absolute value but the difference in energy between the easy and hard directions.

\section{EFFECTIVE DISCRETENESS}

Beginning with an energy Hamiltonian that includes terms for the exchange and anisotropy, we will obtain a solution similar to Paul [8]. Substituting this solution back in to the energy formulation of each atom and summing gives a mathematical series, one term of which can be physically interpreted as the energy arising from the discreteness of the lattice. This is where the moving domain wall feels the effect of individual atoms instead of the bulk continuum potential that has heretofore been so successful in describing materials with much wider walls. 
Egami and Graham [10] have noted that "the potential barrier can be expressed as a single sinusoidal function of the position of the wall, with the periodicity of the interlayer spacing," and determined that true energy minimization always leads to a reasonably narrow wall being centered between atoms, rather than evenly on top of those sources of potential.

The energy Hamiltonian on each atom is equal to

$$
\Gamma_{i}=A\left(\frac{\Delta \theta}{\Delta x_{i}}\right)^{2}+K\left(\sin \theta_{i}\right)^{2}
$$

where $A$ is the coefficient of the nearest-neighbor exchange and $K$ is the generalized anisotropy. A differential change of magnetization angle with respect to position is inappropriate, since the limit cannot be taken with the distance between points approaching zero. The units on both terms are in volumetric energy density, not areal density as is sometimes reported. It becomes more relevant in our case to consider the total volume between atoms, when an areal density would only have accounted for one value at each atom and not through the space between.

Finding the minimum of the similar Euler equation with respect to $\theta$ by setting the variation in $\Gamma$ equal to 0 gives

$$
\theta_{i}=\arcsin \left(\operatorname{sech} \frac{x_{i}-x_{0}}{\delta}\right)
$$

where the derived characteristic length $\delta=\sqrt{A / K}$. The wall width, or the region over which the majority of the magnetic rotation takes place, can be taken as $\pi \delta$. The shift factor $x_{0}$ accounts for a wall centered other than at the origin. It is also possible to use the equivalent solution presented in Bishop and Lewis [15] of the form atan $(\exp ( \pm \omega x))$, where $\omega$ is some frequency under a Lorentz transformation. However in this work, domain walls are always assumed to be moving at much less than the speed of light. We use boundary conditions to further restrict our consideration to $180^{\circ}$ walls.

Substituting our solution for the differential equation back to the Hamiltonian, it reduces to

$$
\Gamma_{i}=2 K\left(\operatorname{sech} \frac{x_{i}-x_{0}}{\delta}\right)^{2}
$$

We employ Poisson's sum formula to sum over all space:

$$
\sum_{i} \Gamma_{i}=\sum_{n} 2 K\left(\operatorname{sech}\left(\frac{(n+\epsilon) l}{\delta}\right)\right)^{2}=\sum_{s} \int_{-\infty}^{\infty} \Gamma(n) \cos (2 \pi s n) d n
$$

The offset $\epsilon$ is added to allow for a non-integer equilibrium position of the domain wall as the basis by which to take the derivative when converting between energies and forces. We change position variables to a temporary placeholder $p=(n+\epsilon) l, d p=l d n$, noting the cancellation of odd terms in an integration over all space, and obtain an expression for the total energy of the domain wall as

$$
\sum_{s} \frac{4 \pi^{2} s K \delta^{2}}{l^{2} \sinh \left(\frac{\pi^{2} s \delta}{l}\right)} \cdot \cos 2 \pi s \epsilon
$$

where the $s=0$ term gives the expected continuum contribution, known to be $\frac{4 \sqrt{A K}}{l}$, and the $s=1$ term gives

$$
\frac{4 \pi^{2} K \delta^{2}}{l^{2} \sinh \left(\frac{\pi^{2} \delta}{l}\right)} \cdot \cos 2 \pi \epsilon
$$




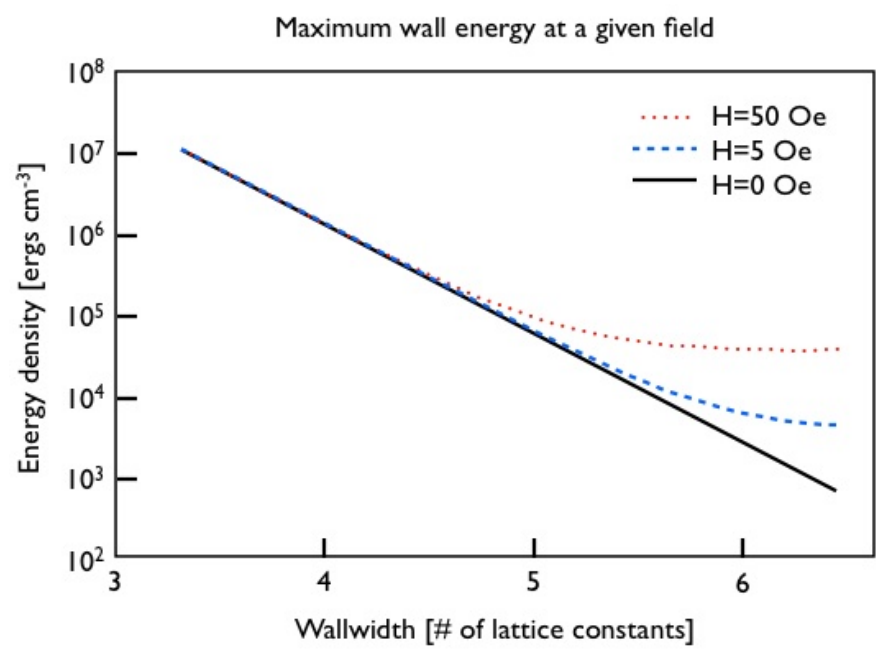

FIG. 1: The cutoff between energies considered "high" and negligible is taken where the lines converge, or where the magnitude of the field becomes less important than the potential inherent in the lattice. The Hamiltonian is on the ordinate axis and includes terms for applied field, anisotropy, and exchange, but excludes time-dependence.

which can be interpreted as the energy arising from the intrinsic potential that a domain wall must overcome in order to move. The $s>1$ terms are vanishingly small because of the hyperbolic sine in the denominator.

It is evident from the pairing of $\delta$ and the lattice spacing $l$ that it is the relative and not the absolute wall width which has any bearing on the transition between a material that is well approximated by the continuum model and a material that requires higher order intrinsic terms. For a domain wall of less than about four lattice constants in width, the continuum approximation deviates significantly from that where the atomic potential is considered. This deviation is shown in Figure 1.

We determine the coercivity due to the discreteness and find it to be substantial below a wall width of about 4 unit cells. Coercivity is defined here as the maximum field in which there are static solutions to the problem, i.e., the position of the wall does not tend to infinity. This is equivalent to finding the field required to make the Zeeman term of $M \cdot H$ equal in magnitude to the intrinsic lattice energy. It can also be thought of as the maximum depth of the potential well due to the atoms. Any external field with magnitude less than the coercivity will instead have the effect of starting the wall oscillating within the atomic potential well. In some highly abstract sense, the coercivity can be thought of as showing the mass, or inertial strength of the wall. Kittel and Galt [16] showed this to be inversely proportional to the thickness of a wall in a continuum model. For a similar treatment of dislocations in metals, see also Nabarro [7].

Expanding, the Hamiltonian is now defined as

$$
\Gamma_{i}=A\left(\frac{d \theta}{d x_{i}}\right)^{2}+K\left(\sin \theta_{i}\right)^{2}-H M_{i} \cos \theta_{i}
$$

Summing over all space in a $2 \pi$ periodic medium and taking the derivative with respect to the dimensionless $\epsilon$ representing the interatomic position in the argument of the cosine, a minimum coercivity due to discreteness is 


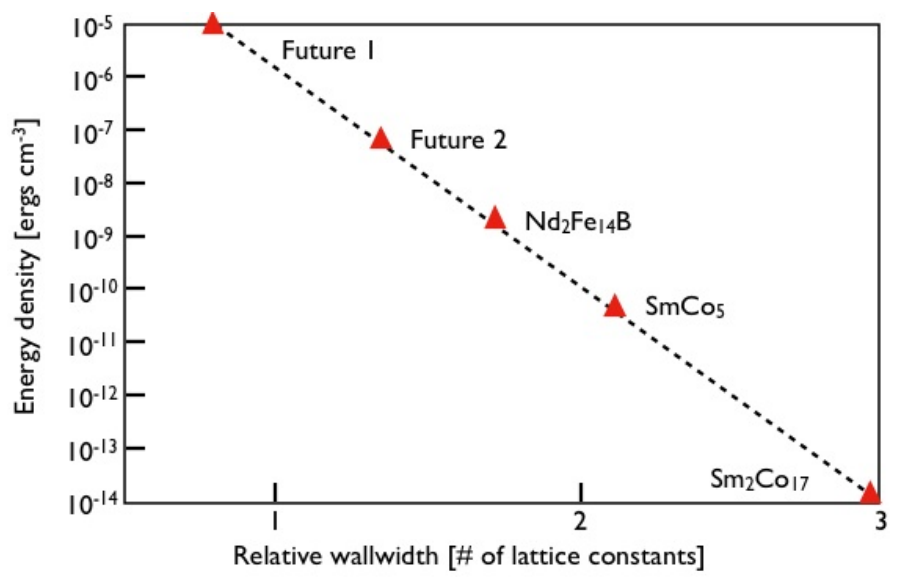

FIG. 2: Intrinsic coercivity as a function of relative wallwidth, with remarkable adherence to a simple linear fit. Recall that the anisotropy and exchange parameters of Future 1 and Future 2 were chosen in a self-consistent manner to be within a factor of 3 of currently available materials.

obtained as

$$
H_{c}=\frac{8 \pi^{3} K\left(\frac{\delta}{l}\right)^{2}}{M \sinh \left(\frac{\pi^{2} \delta}{l}\right)}
$$

The fit of experimental data to the following empirical equation is remarkable.

$$
H_{c}=10^{6} \times \exp \left(\frac{-4 \pi}{3} \cdot \frac{\delta}{l}\right)
$$

For comparison, consider the poor fitting of the energies to a line based on what is typically considered to be the sole dominant parameter, the absolute wall width, without correcting for the lattice parameter of the material (Figures 3 and 4). An intriguing feature of this plot is the relative magnitudes of neodymium-iron-boron and $\mathrm{SmCo}_{5}$. The samarium-cobalts have long been considered ferromagnetically harder than $\mathrm{Nd}_{2} \mathrm{Fe}_{14} \mathrm{~B}$, so we confirm that indeed the relative wall width over lattice spacing is the dominant parameter. This description is not meant to include materials with different processing history where domain wall pinning is the primary mechanism of coercivity.

\section{DEFECT INTERACTION}

In previous sections we only considered domain walls moving in an infinite sinusoidal medium. In this part we discuss the static solutions to a narrow wall in a medium with a planar defect of a certain width, and compare these solutions to those obtained using a continuum picture. Low-dimensional precipitation defects such as those discussed by Kersten are neglected [17]. In brief, a precipitation defect is a defect above some critical size where the additional energy from the interface with the defect is balanced by the reduction in energy coincident with the decreased total length of the domain wall. It can be assumed here that there will be a functionally similar critical size faced by defects in a medium containing very narrow magnetic walls.

Following Paul and Quattrochi [6], space is divided into three regions, two infinite perfect grains of anisotropy angles perpendicular to the x-axis and a defect region of inconsistent properties, as in Figure 5. In order to isolate the effects 


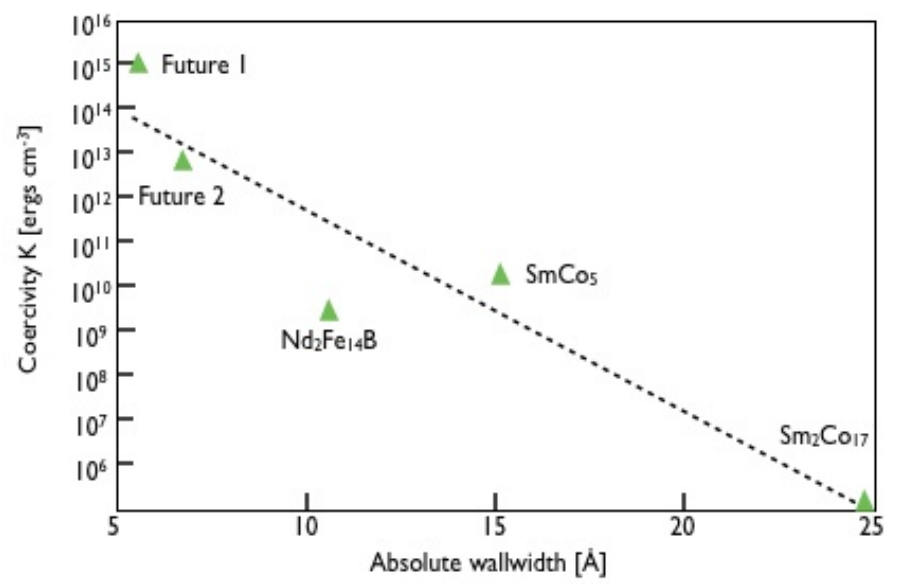

FIG. 3: Intrinsic coercivity as a function of absolute wallwidth fits more poorly to a linear extrapolation. It is informative to notice that the absolute wallwidth of $\mathrm{Nd}-\mathrm{Fe}-\mathrm{B}$ is less than that of $\mathrm{SmCo}_{5}$, since it is considerably less magnetically hard.

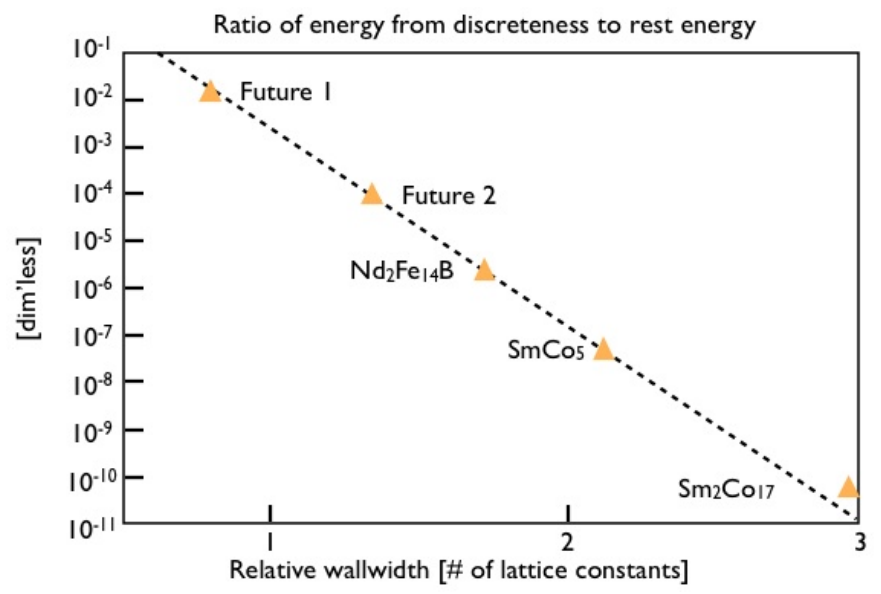

FIG. 4: Relative magnitude of the coercivity and the total wall energy with contributions from the boundary conditions and the intrinsic term, plotted against the relative wallwidth.

of magnetic changes, we again restrict our discussion to $180^{\circ}$ walls. In addition to complicating the influence of the narrowness of the wall, a change in anisotropy angle any amount other than $180^{\circ}$ requires yet another discontinuity in the axial properties. The approximation here is for an abrupt interface at the defect, mimicking a vertically integrated heterostructure with three-dimensional epitaxy, for example, instead of a Gaussian distribution of the defect for a less well-defined inclusion or grain boundary.

The Hamiltonian is defined as before, with the subscripts $j$ indicating the region

$$
\Gamma_{i}=A_{j}\left(\frac{\Delta \theta}{\Delta x_{i}}\right)^{2}+K_{j}\left(\sin \theta_{i}\right)^{2}-H M_{j} \cos \theta_{i}
$$

Three integral equations are now formulated, with

$$
\Gamma_{R I}=\sum_{-\infty}^{x_{1}} \Gamma_{i}
$$




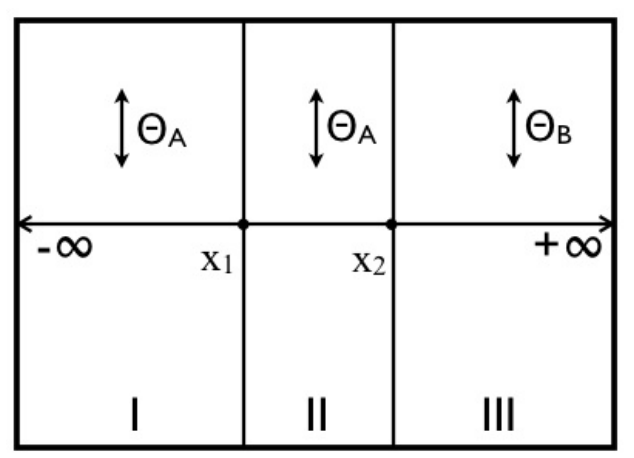

FIG. 5: Regions I and III are considered perfect grains, differing only in the boundary value of the direction of magnetization but having the same anisotropy, exchange, and saturation magnetization value. The external field is applied parallel to the anisotropy axis.

$$
\begin{gathered}
\Gamma_{R I I}=\sum_{x_{1}}^{x_{2}} \Gamma_{i} \\
\Gamma_{R I I I}=\sum_{x_{2}}^{+\infty} \Gamma_{i}
\end{gathered}
$$

Continuity at the defect boundaries is imposed by requiring $\theta$ and $A_{j} \theta^{\prime}$ to be equal. Manipulating the result confirms the fully general expression presented in Paul and Quattrochi [6].

$$
b\left(\cos \theta_{1}\right)^{2}+h a\left(\cos \theta_{1}\right)-b\left(\cos \theta_{2}\right)^{2}-h a\left(\cos \theta_{2}\right)=0
$$

The dimensionless parameters $a, b$, and $h$ are the same as in the reference: $a=\left(\frac{A_{1} M_{1}}{A_{2} M_{2}}\right)-1, b=\left(\frac{A_{1} K_{1}}{A_{2} K_{2}}\right)-1$, and normalized field $h=\frac{M_{2} H}{K_{2}}$. The integral equation that must then be solved is

$$
\int_{x_{1}}^{x} \frac{d x}{\delta_{2}}=\int_{\theta_{1}}^{\theta} d \theta\left((\sin \theta)^{2}-h \cos \theta+b\left(\sin \theta_{1}\right)^{2}-h a \cos \theta_{1}+h(a+1)\right)^{-\frac{1}{2}}
$$

The relative defect width $w=\frac{x_{2}-x_{1}}{\delta_{2}}$ is found when $x$ is set equal to $x_{2}$ in the integral, and is normalized by the projected domain wall halfwidth in the defect region, $\delta_{2}=\sqrt{\frac{A_{2}}{K_{2}}}$.

Combinations of $\theta_{1}, \theta_{2}$, and $h$ consistent with a static solution to the problem were calculated. Figure 6 shows the correlation between increasing change in magnetization angle over the defect with increasing applied field for $a$ and $b$ values corresponding to approximately a ten per cent change across the defect region. For each increase in Zeeman energy, the material concentrates more of the rotation in the defect region.

Figure 7 uses the material parameters of $\mathrm{SmCo}_{5}$ and the same values of defect strength to show an example of defect behavior in a real material. Note that the estimation by integration of the energy of interaction between a domain wall and a defect is always lower by a factor of two. This is most true at the narrowest defect widths, tapering as the effect of individual atoms is smoothed out over a wider imperfection in the crystal.

In high strength defects $a=b=0.5$ (not shown), the energy calculation based on summation produces a smaller result than that based on integration. Exchange is a correlation term that affects not just the atom in question but 


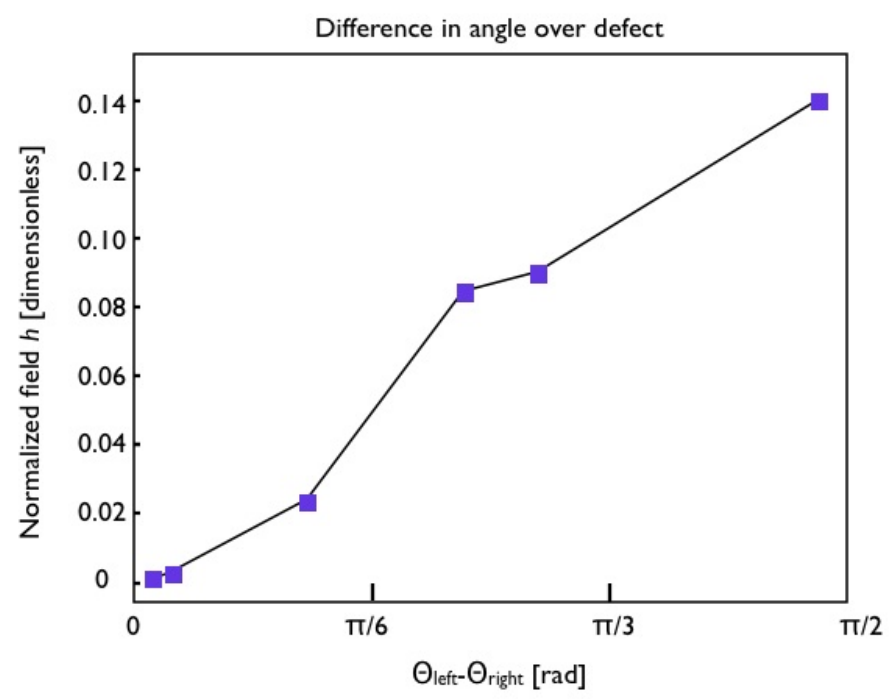

FIG. 6: Monotonically increasing change in magnetization angle across the defect with increasing applied external field. This shows that for increasing magnetic field energy, the material concentrates more rotation in an already imperfect region, maximizing the volume of the medium that can be considered perfect. $a=0.1, b=0.294$ corresponds to a $10 \%$ change in magnetic properties across the defect region as compared to the bulk.

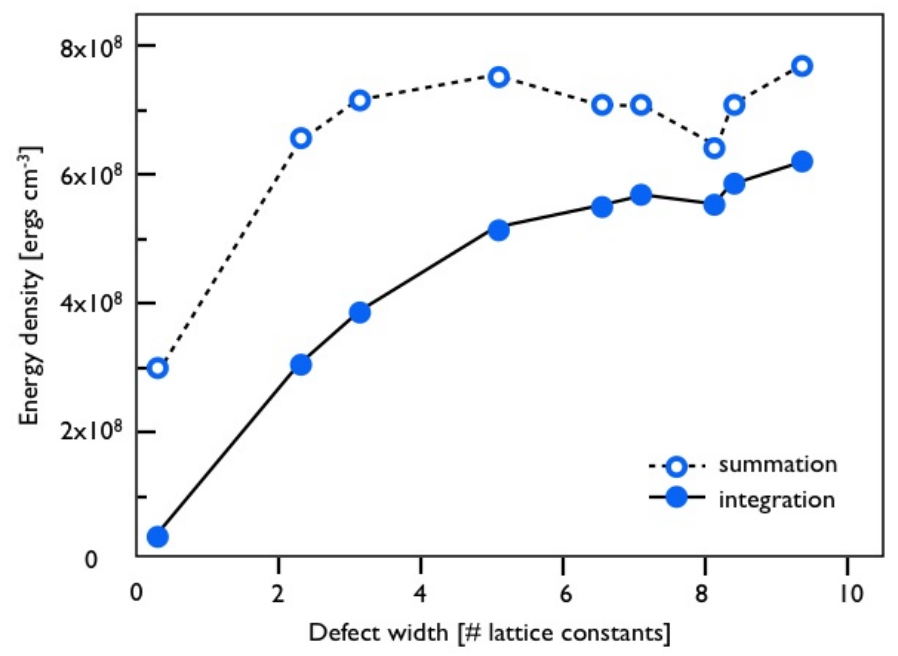

FIG. 7: Relative heights of energy calculations from integration and summation. The estimation of wall energy at a given defect width from discreteness is always larger in a relatively weak defect than from integration.

its neighbors, which means that even the narrowest defect will jog the equilibrium of both the domain wall shape and its position: at minimum, one to two planes of atoms are forced to an unstable state, which has more of a direct effect on the terms in the summation. 


\section{TIME DEPENDENCE}

In order to solve for the time dependence of an ultra-narrow ferromagnetic domain wall moving in a perfect crystal, a pseudo-viscosity term is now added to the energy Hamiltonian and the slight perturbation in the argument of $\theta$ is defined to have a time-varying part. For a thorough treatment of the mathematics in this section refer to Titchmarsh [18] or Morse and Feshbach [19].

The form of the unperturbed domain wall can be recognized mathematically as a soliton wave, explained succinctly in Nakajima [20]. The most important aspect of a true soliton wave is that it retains its shape during propagation, neither compressing nor distorting. The definition over all space of the energy now includes a wall pseudo-viscosity term, which can be physically intuited as representing eddy currents (Kittel and Galt [16]), demagnetization fields from time-lagged precession of spins (Boutron [21]), and/or microdefect interactions (Kittel [13]).

The equation of small-amplitude motion of a $180^{\circ}$ wall has previously been described in a linear model of the form

$$
m \ddot{z}+\beta \dot{z}+\alpha z=2 I H
$$

where $m$ is the mass of the wall, $\beta$ is the pseudo-viscosity, and $\alpha$ is fairly arbitrary lattice stiffness chosen to make the equations work out. The right-hand side of the equation is the pressure on the wall and $H$ is the applied field. In large enough fields to overcome the atomic potential the inertial term becomes negligible and the equation reduces to a differential of first order.

Our total Hamiltonian in a perfect lattice becomes

$$
A\left(\frac{\delta \theta}{\delta x_{i}}\right)^{2}+K\left(\sin \theta_{i}\right)^{2}-M H \cos \theta_{i}-\eta\left(\frac{\delta \theta_{i}}{\delta t}\right)-C\left(\frac{\delta \theta_{i}}{\delta t}\right)^{2}=\Gamma_{i}
$$

where the angle of magnetization $\theta$ is no longer a direct function of the wall position $x+\epsilon$ but is instead $\theta \approx \theta_{0}+\epsilon \theta^{\prime}$, where the second term is written as $\phi$. The units on the constant $C$ must be [length $]^{2}$ because $\theta$ is dimensionless. The coefficient on the prime terms includes a Legendre polynomial as a function only of $\left(-\tanh \frac{x}{\delta}\right)$ and a time-dependent solution only of time $T(t)$.

$$
A\left(\frac{d \theta_{0}}{\delta x_{i}}+\frac{d \phi}{\delta x_{i}}\right)^{2}+K \sin \left(\theta_{0}+\phi\right)^{2}-M H \cos \left(\theta_{0}+\phi\right)-\eta\left(\frac{d \phi}{d t}\right)-C\left(\frac{d \phi}{d t}\right)^{2}
$$

The intrinsic potential is included in the manipulations of the term $\theta_{0}$. We restrict the solution to the first order polynomials

$$
\phi=P_{\text {total }} T_{\text {total }} \quad P_{1}^{1}=\frac{l}{\delta} \operatorname{sech} \frac{l \epsilon}{\delta}
$$

Higher order polynomials $P_{1}^{i \mu}$ are ignored as representing radiative spin waves, which are also included abstractly in the definition of $\eta$. Projecting out, only terms of the first kind remain, the orthogonality of higher order polynomials with each having cancelled the rest. The assumption is made that $\phi$ is of a size that the small angle sine approximation can be made. The second order differential in time results:

$$
\ddot{T}+\frac{\eta}{2 C} \dot{T}=\frac{\pi^{2} A}{C l^{2} \sinh \frac{\pi^{2} \delta}{l}}+\frac{H M}{2 C}
$$




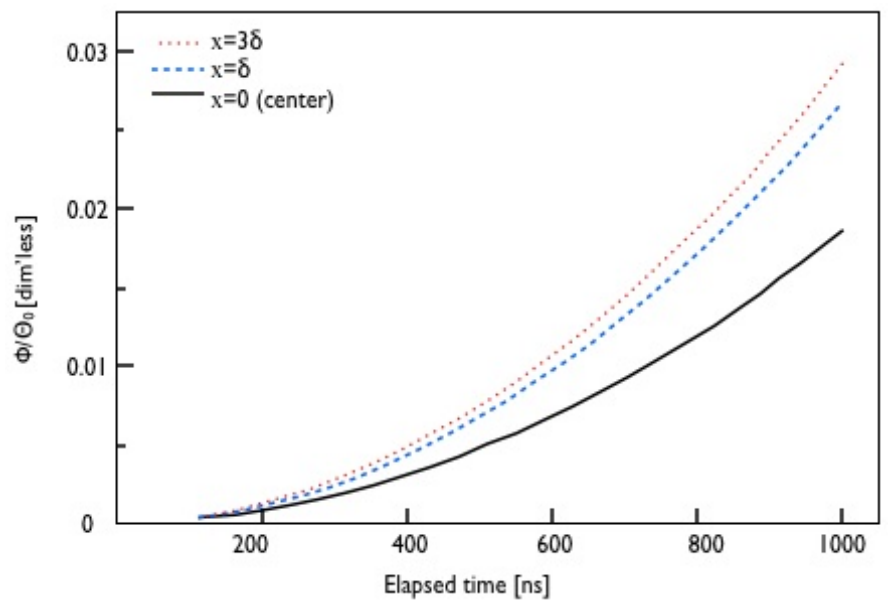

FIG. 8: Relative magnitude of the perturbation compared with the size of the equilibrium angle distribution at a given point. This confirms our assumption that it is small and allows us to make some necessary simplifications in the integration.

This obeys boundary conditions $T=\dot{T}=0$ at $t=0$ and the canonical solution

$$
T=s \exp \left[\frac{-t}{n}\right]+\frac{s}{n} t-\frac{s}{n}
$$

where $n=\eta /(2 C), p=\delta / l$, and $s=\frac{2 \pi^{2} K p^{2}}{\eta \sinh \left(\pi^{2} p\right)}+\frac{H M}{\eta}$.

From this expression it can be seen that at some time large enough, the perturbation $\epsilon$ is no longer small. However, the wall would not experience this condition in a real crystal with average dislocation density of $10^{4} \mathrm{~cm}^{-2}$, where the wall would not travel unhindered for long enough. It can be seen in Figure 8 that the size of the perturbation is small for all relevant times on the scale of a material response to a magnetic field by comparing the size of $\epsilon / \theta_{0}$ at $x=0$.

Figure 9 models the effect of an increasing intrinsic $\eta$ (representing eddy currents, spin waves, etc.) on the motion of the wall at any given time. Only the T part of the perturbation is plotted, because the maximum distortion from the equilibrium distribution with time is felt at the midpoint of the wall because of the hyperbolic secant coefficient, which falls off to zero away from the center. Figure 10 shows the motion of the defined center of the wall affected by single atomic potentials. Larger $\eta$ begins to smooth out the effects from a single atom and shows only the cumulative consequence of damped motion.

\section{CONCLUSIONS}

The effects of atomic discreteness on the behavior of ultra-narrow ferromagnetic domain walls were explored analytically. The important result that it is the relative wallwidth and not the absolute wallwidth is presented as it arises clearly from the lowest order terms in the solution to the energy equations. In the presence of a relatively weak defect, it is found that the energy in an ultra-narrow wall will always be greater than would have been predicted by a continuum model. Time-dependent solutions to the equations of motion for these domain walls of interest were found for varying values of the effective viscosity. 


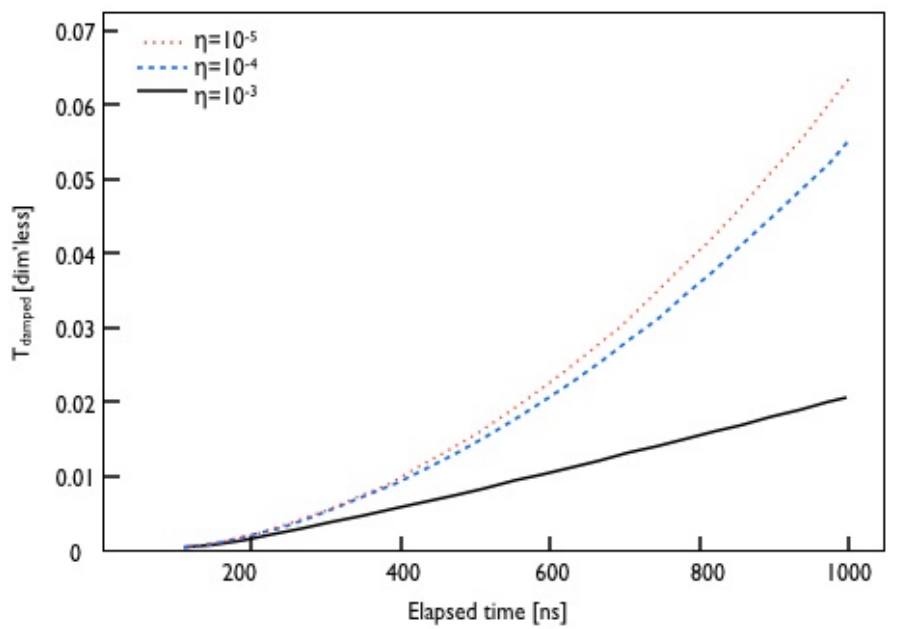

FIG. 9: Showing the effect of increasing viscosity $\eta$ on the ability of the center of a domain wall to respond to a small applied field of $\mathrm{H}=20$ Oe. No static solutions are found for $\eta=0$; an infinitesimally small field applied to a domain wall in a perfect crystal will set it moving forever.

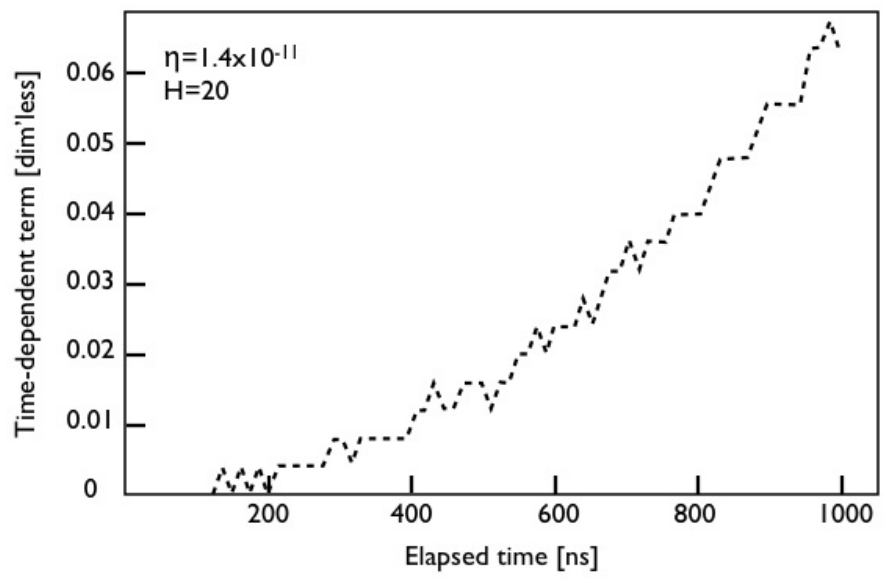

FIG. 10: Proposed to show the effects of individual atoms on the movement of the domain wall in a medium with small $\eta$, increasing the relative effect of the intrinsic potential on the equation of motion of the wall.

Natural magnetic materials with ultra-high anisotropy and synthetic magnetic nanostructures with threedimensional epitaxy require such narrow-wall analysis as presented here. As materials processing catches up with the ability of mathematics to assert perfect crystals and ultra-narrow walls, the continuum approximation of matter will break down, and will do so significantly. Engineering defects to known widths and strengths will pin narrow walls reliably under appropriate field conditions, which can be exploited in high-density magnetic recording and other applications. 


\section{Acknowledgements}

Work at the ALS was supported by the Director, Office of Basic Energy Sciences, Department of Energy, under contract No. DE-AC02-05CH11231.

[1] The ferromagnetic shape-memory effect in NiMnGa, MA Marioni, RC O'Handley, SM Allen, SR Hall, DI Paul, ML Richard, J Feuchtwanger, BW Peterson, JM Chambers, and R Techapiesancharoenkij, J. Magn. Magn. Mat., vol. 290-291 (2005), pp35-41.

[2] Theory of magnetization: Twin boundary interaction in ferromagnetic shape memory alloys, DI Paul, J. Marquiss, and D. Quattrochi, J. Appl. Phys. 93, vol. 8 (2003), pp4561-4565.

[3] Ferromagnetic shape memory alloys: theory of interactions, DI Paul, RC O'Handley, and BW Peterson, J. Appl. Phys., $9710 \mathrm{M} 312(2005)$.

[4] Ferromagnetic shape memory alloys: a theoretical approach, DI Paul, W McGehee, RC OHandley, and M Richard, J. Appl. Phys. 101, 123917 (2007).

[5] Dynamics of sine-Gordon solitons in the presence of perturbations, MB Fogel, SE Trullinger, AR Bishop, JA Krumhansl, Phys. Rev. B 15 vol. 3 (1977) pp1578-1592.

[6] Magnetization distribution and coercivity in multigrain materials, DI Paul and D Quattrochi, J. Appl. Phys. 91 vol. 10 (2002).

[7] Dislocations in a simple cubic lattice, FRN Nabarro, 1946.

[8] Soliton theory and the dynamics of a ferromagnetic domain wall, DI Paul, J. Phys. C, vol. 12 (1977).

[9] Über die trägheit der Wände zwischen Weißchen Bezirken, W Döring, Z. Naturforsch., 3a, 373 (1948).

[10] Domain walls in ferromagnetic Dy and Tb, T. Egami and CD Graham, J. Appl. Phys. 42 vol. 4 (1971), pp1299-1300.

[11] Dynamics and statistical mechanics of a one-dimensional model Hamiltonian for structural phase transitions, JA Krumhansl and Schrieffer, Phys. Rev. B 11, vol. 9, (1975).

[12] Ferromagnetic Materials (vol. 4), K.J. Strnat, ed. E.P. Wohlfarth and K.H.J. Buschow, pp 131-209, (1988).

[13] Physical theory of ferromagnetic domains, C Kittel, Rev. Modern Phys., 21 vol. 4, (1949).

[14] Die Dynamik der Blochschen Wand, U Enz, Helvetia Physica Acta, vol. 37 (1964).

[15] A theory of intrinsic coercivity in narrow magnetic domain wall materials, AR Bishop and Lewis, J. Phys. C 12, 1979, p3811.

[16] Advances in Solid-State Physics, vol. 3 Ferromagnetic domain theory, C Kittel and J Galt, pp534-564.

[17] Grundlagen einer neuen Theorie der Koerzitivkraft, M Kersten, Leipzig, 1943.

[18] The Theory of Functions, 2nd ed., EC Titchmarsh, Oxford, 1947.

[19] Methods in Theoretical Physics, P Morse and H Feshbach, McGraw-Hill, New York, 1953.

[20] Nonequilibrium stationary coupling of solitons, K Nakajima, Y. Sawada, and Y Onodera, J. Appl. Phys. 46 (1979), pp52725279 .

[21] Facteur de transmission d'une paroi de Bloch pour des ondes de spin de vecteur d'onde normal a la paroi, F Boutron, Academie des Sciences, (1961) pp. 3955-3957. 